\title{
Erratum to: Chronic hyperglycemia affects bone metabolism in adult zebrafish scale model
}

\author{
Marta Carnovali $^{1}$ Livio Luzi ${ }^{2,3}$ - Giuseppe Banfi ${ }^{4,5} \cdot$ Massimo Mariotti $^{4,6}$
}

Published online: 11 April 2017

(C) Springer Science+Business Media New York 2017

Erratum to: Endocrine (2016) 54:808-817 DOI 10.1007/s12020-016-1106-3

The co-author Giuseppe Banfi is affiliated with Vita-Salute San Raffaele University and IRCCS Galeazzi. But in the original version of this article, IRCCS Galeazzi is unfortunately not included as author affiliation. The error in author affiliation is corrected with this erratum.

The online version of the original article can be found at doi:10.1007/ s12020-016-1106-3.

$\triangle$ Massimo Mariotti massimo.mariotti@unimi.it

1 Gruppo Ospedaliero San Donato Foundation, Milan, Italy

2 Policlinico San Donato IRCCS, Milan, Italy

3 Department of Biomedical Sciences for Health, University of Milan, Milan, Italy

4 IRCCS Galeazzi Orthopedic Institute, Milan, Italy

5 Vita-Salute San Raffaele University, Milan, Italy

6 Department of Biomedical, Surgical and Dental Sciences, University of Milan, Milan, Italy 